\title{
Optical-thermal mathematical model for endovenous laser ablation of varicose veins
}

\author{
Peter W. M. van Ruijven • Anna A. Poluektova • \\ Martin J. C. van Gemert • H. A. Martino Neumann • \\ Tamar Nijsten • Cees W. M. van der Geld
}

Received: 18 September 2013 / Accepted: 22 September 2013 /Published online: 9 October 2013

(C) Springer-Verlag London 2013

\begin{abstract}
Endovenous laser ablation (EVLA) is successfully used to treat varicose veins. However, the exact working mechanism is still not fully identified and the clinical procedure is not yet standardized. Mathematical modeling of EVLA could strongly improve our understanding of the influence of the various EVLA processes. The aim of this study is to combine Mordon's optical-thermal model with the presence of a strongly absorbing carbonized blood layer on the fiber tip. The model anatomy includes a cylindrically symmetric blood vessel surrounded by an infinite homogenous perivenous tissue. The optical fiber is located in the center of the vessel and is withdrawn with a pullback velocity. The fiber tip includes a small layer of strongly absorbing material, representing the layer of carbonized blood, which absorbs $45 \%$ of the emitted laser power. Heat transfer due to boiling bubbles is taken into account by increasing the heat conduction coefficient by a factor of 200 for temperatures above $95^{\circ} \mathrm{C}$. The temperature distribution in the blood, vessel wall, and surrounding medium is calculated from a numerical solution of the bioheat equation. The simulations were performed in MATLAB $^{\mathrm{TM}}$ and validated with the aid of an analytical
\end{abstract}

P. W. M. van Ruijven • A. A. Poluektova $(\bowtie) \cdot$

C. W. M. van der Geld

Department of Mechanical Engineering,

Eindhoven University of Technology, Eindhoven, The Netherlands

e-mail: ivanova.anna.1987@gmail.com

C. W. M. van der Geld

e-mail: C.W.M.v.d.Geld@tue.nl

M. J. C. van Gemert

Department of Biomedical Engineering and Physics,

Academic Medical Centre, University of Amsterdam,

Amsterdam, The Netherlands

H. A. M. Neumann · T. Nijsten

Department of Dermatology, Erasmus Medical Centre,

Rotterdam, The Netherlands solution. The simulations showed, first, that laser wavelength did virtually not influence the simulated temperature profiles in blood and vessel wall, and, second, that temperatures of the carbonized blood layer varied slightly, from 952 to $1,104{ }^{\circ} \mathrm{C}$. Our improved mathematical optical-thermal EVLA model confirmed previous predictions and experimental outcomes that laser wavelength is not an important EVLA parameter and that the fiber tip reaches exceedingly high temperatures.

Keywords Endovenous laser ablation (EVLA) · Carbonized blood layer $\cdot$ Light transport $\cdot$ Heat transfer $\cdot$ Heat generation

\section{Introduction}

Endovenous laser ablation (EVLA) is an efficient method to treat insufficient varicose veins as it has high occlusion rates with few complications. Postoperatively, patients often complain of a burning sensation around the vein. That is why many studies addressed the thermal aspects of EVLA and its side effects.

EVLA procedures involve a number of steps. First, a hollow catheter is introduced in the varicose vein of the patient followed by advancing an optical laser fiber through the catheter. In great saphenous veins, the fiber is advanced to a few centimeters away from the cross, where the saphenous and femoral veins join together. Next, tumescent anesthetic fluid at room temperature of about $20^{\circ} \mathrm{C}$ is administered at increased pressure to the perivenous tissue surrounding the varicose vein. Importantly, this fluid forces the vein wall to be folded tightly around the catheter, and provides cooling to the perivenous tissue. Subsequently, the laser is switched on, and the fiber emitting the laser light is pulled back simultaneously. The emitted laser light is scattered and absorbed by the blood and vein wall, resulting in heat generation. Finally, this generated heat induces a temperature rise of the blood and the vein wall, which triggers occlusion mechanisms and eventually leads to obliteration of the vein. 
Revealing the mechanisms of action of EVLA is a prerequisite to find optimal treatment parameters (i.e., laser power $P$ (watts), pullback velocity $v$ (millimeters per second), and laser wavelength $\lambda$ (nanometers)) and to further improve the clinical results. However, despite many studies [1-11], the exact working mechanisms are still not fully identified.

To improve our understanding of the ongoing processes during EVLA, Mordon et al. developed the first mathematical optical-thermal EVLA model based on propagation of laser light through absorbing and scattering tissues (blood and vessel wall), heat generation due to light absorption, conductive heat transfer, and tissue damage in the vein wall by a first order Arrhenius equation [8]. These authors did not incorporate the thin layer of strongly absorbing carbonized blood that always develops on the fiber tip, as this was described after they published their model [12]. Most likely, this black layer is the cause of the observed exceedingly high temperatures $[1,4$, $9,10]$, which have been suggested to contribute to vein wall injury [11]. Our aim, therefore, is to combine Mordon's model with the presence of a strongly absorbing carbonized blood layer on the fiber tip. For simplicity, we only simulated temperature profiles and did not include the Arrhenius damage integral.

\section{Methods}

The mathematical model describes the following cascade of physical processes occurring during EVLA: light transport, heat generation, and heat transfer.

\section{Geometry}

The anatomy of the fiber probe, assumed to be centered in the middle of the vein, and coordinate system $(r, z)$ are presented in Fig. 1. Light emission is assumed to be spherical symmetric and centered at the top of the fiber probe that moves in axial direction, $z$, with typical velocity $v=2 \mathrm{~mm} / \mathrm{s}$. In the domain $\{0$ $<z<L=200 \mathrm{~mm}, 0<r<R=10 \mathrm{~mm}\}$, in the laboratory system in which the vein is at rest, $L$ and $R$ are boundaries imposed by the computational domain. Four different volume zones are distinguished as follows: laser fiber (I), lumen of the vein (II), vein wall (III), and surrounding perivenous tissue (IV). The inner radius of the vein $r_{i}$ is taken equal to $1.5 \mathrm{~mm}$ and the outer radius $r_{0}$ is $2.5 \mathrm{~mm}$. At time zero, the probe tip is at $(0$, $\left.z_{0}=20 \mathrm{~mm}\right)$ and at time $t$, it is at $\left(0, z=z_{0}+v \cdot t\right)$. The spherical radius from the fiber tip is defined as follows:

$r_{s}=\sqrt{r^{2}+\left(z-z_{0}-\mathrm{v} \cdot t\right)^{2}}$,

Where $(z, r)$ indicates an arbitrary location in the domain. In the present work, we will evaluate temperatures at axial coordinate $z=0.2 \cdot L=40 \mathrm{~mm}$.

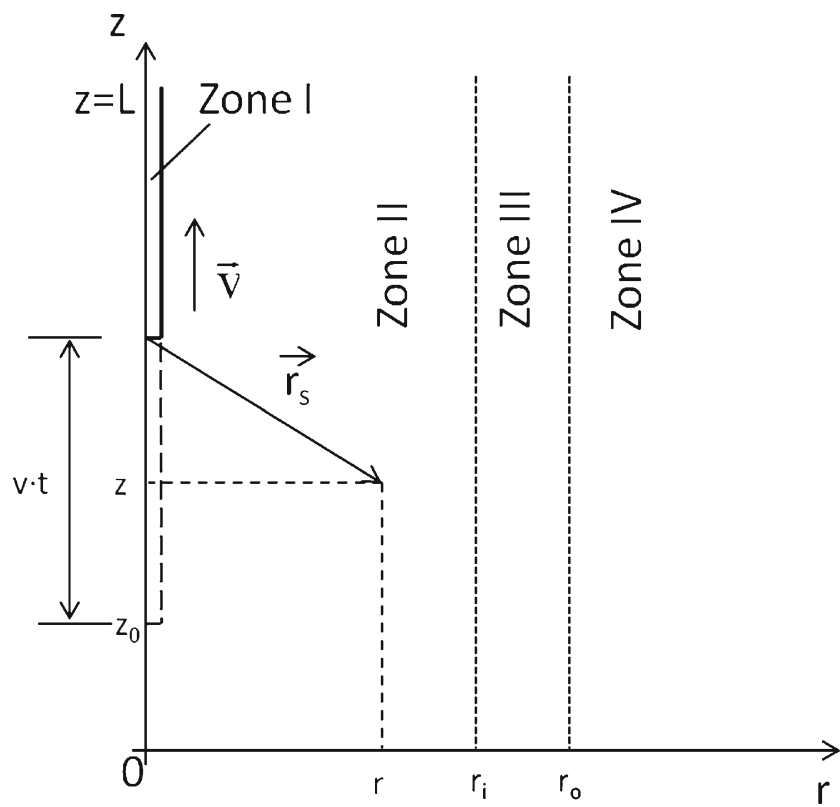

Fig. 1 Geometry of vein and fiber with $r_{i}$ and $r_{o}$ the inner and outer radius of the vein: laser fiber (zone I), lumen of the vein (zone II), vein wall (zone III) and surrounding perivenous tissue (zone IV). Not to scale

\section{Light transport}

The light power emitted out of the fiber tip into the ambient blood is scattered towards the vein wall and the surrounding tissue. We recently showed that $45 \pm 10 \%$ of the supplied light is absorbed in a thin and highly-absorbing layer of carbonized blood deposited on the fiber tip [12]. The remaining $55 \%$ of the light penetrates into the blood, vein wall, and surrounding tissue. The model used for light transport is the diffusion approximation of the theory of radiative transfer from an isotropic point source, which is an appropriate approximation here because of the highly scattering character of blood and vein wall. The fluence rate $\phi$, as a function of the spherical radius from the fiber tip, $r_{s}$, is given as follows [13]:

$\phi\left(r_{s}\right)=\frac{(1-0.45) \cdot P}{4 \pi D_{d i f} r_{s}} \cdot \exp \left(-\mu_{e f f} r_{s}\right)$,

Where $P$ is the power of the supplied light. The diffusion constant $D_{\text {dif }}$ and effective attenuation coefficient $\mu_{\text {eff }}$ are given as follows:

$$
\begin{gathered}
D_{\text {dif }}=\frac{1}{3\left(\mu_{s}^{\prime}+\mu_{a}\right)}, \\
\mu_{\text {eff }}=\sqrt{3 \mu_{a}\left(\mu_{s}^{\prime}+\mu_{a}\right)}, \\
\mu_{s}^{\prime}=\mu_{s}(1-g),
\end{gathered}
$$

Where $\mu_{a}$ is the absorption coefficient, $\mu_{s}^{\prime}$ the reduced scattering coefficient, $\mu_{s}$ the scattering coefficient, and $g$ the average cosine of the scattering angle (the anisotropy factor), 
Table 1 Thermal properties per zone (see Fig. 1)

\begin{tabular}{llll}
\hline & Unit & $\begin{array}{l}\text { Zone II } \\
\text { Blood }\end{array}$ & $\begin{array}{l}\text { Zone III and IV } \\
\text { Vein wall and perivenous tissue }\end{array}$ \\
\hline$k$ & $\left(\mathrm{~W} / \mathrm{m}^{\circ} \mathrm{C}\right)$ & 0.6 & 0.56 \\
$\rho$ & $\left(\mathrm{kg} / \mathrm{m}^{3}\right)$ & 1,000 & 1,050 \\
$c_{p}$ & $\left(\mathrm{~J} / \mathrm{kg}^{\circ} \mathrm{C}\right)$ & 4,181 & 3,780 \\
$\alpha=k /\left(\rho \cdot c_{p}\right)$ & $\left(\mathrm{m}^{2} / \mathrm{s}\right)$ & $1.43 \cdot 10^{-7}$ & $1.41 \cdot 10^{-7}$ \\
\hline
\end{tabular}

all being a function of medium and wavelength. Equations (1), (2), and (3) can be combined as follows:

$$
\phi(r, \mathrm{z}, t)=\frac{(1-0.45) \cdot P}{4 \pi D_{d i f} \sqrt{r^{2}+\left(\mathrm{z}-\mathrm{z}_{0}-\mathrm{v} \cdot t\right)^{2}}} \cdot \exp \left(-\mu_{\text {eff }} \sqrt{r^{2}+\left(\mathrm{z}-\mathrm{z}_{0}-\mathrm{v} \cdot t\right)^{2}}\right)
$$

\section{Rate of heat generation}

The rate of volumetric heat generation consists of the heat generated in the carbonized layer at the fiber tip, $q_{t i p}$, and heat generated throughout the medium due to absorption of diffused light, $q_{d i f}$. Both $q_{t i p}$ and $q_{d i f}$ are local heat generation densities, in watts per meter [3]. The former term $q_{\text {tip }}$ is described by the ratio of the absorbed power in the thin layer and the layer's volume, $V_{\text {tip }}$. The term $q_{\text {dif }}$ is the product of the local fluence rate and absorption coefficient.

$q_{\text {tip }}=\frac{0.45 \cdot P}{V_{\text {tip }}}$,

$q_{d i f}=\mu_{a}(r, \mathrm{z}) \cdot \phi(r, \mathrm{z}, t)$

As soon as the laser is switched on, the carbonized layer appears. The thickness of the hot layer is about $0.026 \mathrm{~mm}$ [12], and it does not change during the computation.

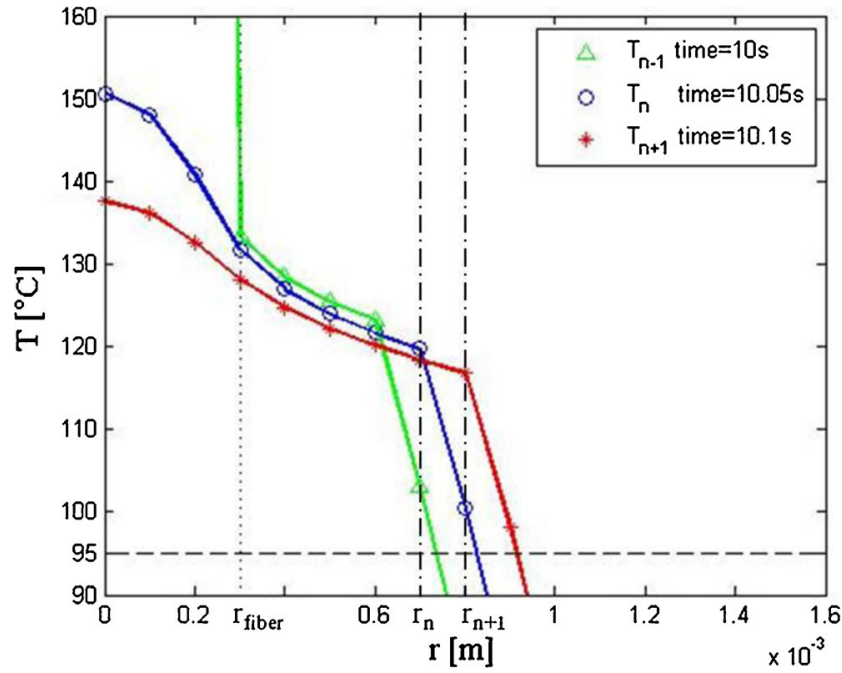

Fig. 2 Temperature profiles at different times depend on the radius, here for $840 \mathrm{~nm}, P=15 \mathrm{~W}, v=2 \mathrm{~mm} / \mathrm{s}$

Heat transfer

Generally, heat transfer in tissue is described by the bioheat equation, which is in fact transient heat conduction, with a source term equal to the absorbed volumetric power, given as follows:

$\rho c_{p} \frac{\partial T}{\partial t}=\nabla \cdot(k \nabla T)+q$,

With the material properties: mass density $\rho$, heat capacity $c_{p}$, and thermal conductivity $k$. In this model, heat transfer by means of convection is not taken into account. The potential impact on heat transfer of this process is acknowledged, but for simplicity, it is disregarded in this study, as well as in Mordon's model [8]. The phase change of blood in the lumen of the vein limits the temperature of the blood to about $100^{\circ} \mathrm{C}$. Boiling is not explicitly simulated, but limiting the maximum temperature in the blood is achieved in the numerical model by enhancement of the local heat conductivity by a factor of 200 if the temperature exceeds a threshold $\left(95^{\circ} \mathrm{C}\right)$. This
Table 2 Optical parameters for venous blood $\left(75 \% \mathrm{HbO}_{2}\right)$, vein wall, and surrounding perivenous tissue

\begin{tabular}{|c|c|c|c|c|c|c|}
\hline \multirow[t]{2}{*}{$\lambda(\mathrm{nm})$} & \multicolumn{3}{|c|}{$\mu_{a}(1 / \mathrm{mm})$} & \multicolumn{3}{|c|}{$\mu_{s}^{\prime}(1 / \mathrm{mm})$} \\
\hline & Blood & Vein wall & Perivenous tissue & Blood & Vein wall & Perivenous tissue \\
\hline 810 & 0.21 & 0.2 & 0.017 & 0.73 & 2.4 & 1.2 \\
\hline 840 & 0.21 & 0.18 & 0.019 & 0.75 & 2.33 & 1.18 \\
\hline 940 & 0.28 & 0.12 & 0.027 & 0.64 & 2.13 & 1.1 \\
\hline 980 & 0.21 & 0.1 & 0.030 & 0.6 & 2.0 & 1.0 \\
\hline 1,064 & 0.12 & 0.12 & 0.034 & 0.58 & 1.95 & 0.98 \\
\hline 1,320 & 0.3 & 0.3 & 0.045 & 0.54 & 1.8 & 0.9 \\
\hline 1,470 & 3.0 & 2.4 & 0.35 & 0.52 & 1.7 & 0.84 \\
\hline 1,950 & 10.0 & 7.5 & 0.35 & 0.52 & 1.7 & 0.84 \\
\hline
\end{tabular}


procedure assures energy conservation (see the Discussion). The threshold temperature of $95{ }^{\circ} \mathrm{C}$ was found by trial and error in order to limit the temperature of the blood to about $100^{\circ} \mathrm{C}$. Superheating of the liquid to temperatures exceeding
$100{ }^{\circ} \mathrm{C}$ only occurs when the combined action of convection (fiber motion) and conduction is insufficient to balance the heat production at the fiber tip.

The mathematical formulation is summarized as follows:

$$
\left\{\begin{array}{lr}
\rho c \frac{\partial T}{\partial t}=\nabla \cdot(k \nabla T)+q(r, z, t) & \text { at }(r, z) \in \Omega, t \geq 0, \\
q=\frac{0.55 \cdot P \mu_{a}}{4 \pi D_{\text {dif }} \sqrt{\left(r^{2}+\left(z-z_{0}-\mathrm{v} \cdot t\right)^{2}\right)}} \exp \left(-\mu_{\text {eff }} \sqrt{\left(r^{2}+\left(z-z_{0}-\mathrm{v} \cdot t\right)^{2}\right)}\right), \\
q\left(z, 0 \leq r \leq r_{\text {fiber }}\right)=\frac{0.45 \cdot P}{V_{\text {tip }}}, & \\
T\left(r_{\text {int }}^{-}, z, t\right)=T\left(r_{\text {int }}^{+}, z, t\right) & \text { if } 0 \leq z \leq L, t \geq 0, \\
\left(k \frac{\partial T}{\partial r}\right)\left(r_{\text {int }}^{-}, z, t\right)=\left(k \frac{\partial T}{\partial r}\right)\left(r_{\text {int }}^{+}, z, t\right) & \text { if } 0 \leq z \leq L, t \geq 0, \\
\frac{\partial T}{\partial t}(r, z, t)=0 & \text { if } r=0 v R, 0 \leq z \leq L, \\
\frac{\partial T}{\partial z}(r, z, t)=0 & \text { if } 0 \leq r \leq R, z=0 v L, \\
T(r, z, 0)=T_{\text {initial }}=293[K] & \text { at } \quad(r, z) \quad \in
\end{array}\right.
$$

Equations (8.1)-(8.3) are supplemented with appropriate boundary and initial conditions in order to be solvable. Essentially, the presence of three different zones implies different optical and thermal parameters for each zone. As a consequence, two internal boundary conditions at each material interface are required to ensure both continuity of temperature $T$, Eq. (8.4), and heat flux $k \frac{\partial T}{\partial r}$, Eq. (8.5), where $r_{i n t}{ }^{-}$and $r_{i n t}{ }^{+}$ are located just left and right of the interface $\left(r_{i}\right.$ and $\left.r_{o}\right)$, respectively. The imposed external boundary conditions naturally follow from the requirement that the boundary follows the adjacent material, resulting in Neumann boundary conditions (8.6), (8.7). Finally, initial condition (8.8) implies it is assumed that the entire domain $\Omega$ is at room temperature at $t=0$.

The various parameters (optical and thermal properties) used are summarized in Tables 1 and 2. The absorption coefficients for blood are derived by assuming a blood hematocrit of $43 \%$, venous blood includes $70 \% \mathrm{HbO}_{2}$, the $\mathrm{HbO}_{2}$ and $\mathrm{Hb}$ absorption spectra are taken from Fig. 2 of Kuenstner and Norris [14], normalized to taking an absorption coefficient of the venous peak at about $760 \mathrm{~nm}$ as $0.9 \mathrm{~mm}^{-1}$ (from optical absorption of hemoglobin by Scott Prahl, through the Internet), and the absorption coefficients for vessel wall and perivenous tissue are taken from Vuylsteke and Mordon [6].

The fiber tip was modeled as a glass tube with thermal conductivity $k_{\text {fiber }}=1.3 \mathrm{~W} / \mathrm{m}^{\circ} \mathrm{C}$.

\section{Model validation}

The constructed model was implemented in MATLAB ${ }^{\mathrm{TM}}$. The implementation was validated by simulating a simplified but comparable situation for which an analytical solution is known. This concerns conduction of heat resulting from a moving isotropic point source (of heat $q$ ) in a uniform medium which is given as follows [15]:

$T=\frac{q}{4 \pi k R} \exp \left(\frac{v \cdot(\mathrm{z}-R)}{2 \alpha}\right) \cdot\left(\frac{1}{2}-\frac{1}{2} \operatorname{erf}\left(\frac{R}{2 \sqrt{\alpha t}}-\frac{v \sqrt{\alpha t}}{2 \alpha}\right)+\frac{\exp \left(\frac{\nu R}{\alpha}\right)}{2} \cdot \operatorname{erfc}\left(\frac{R}{2 \sqrt{\alpha} t}+\frac{v \sqrt{\alpha t}}{2 \alpha}\right)\right)$,

Where $R=\sqrt{r^{2}+z^{2}}$ is the length of radius-vector of current position, erf is the error function, erfc is the complementary error function, and $\alpha=k / c_{p} \cdot \rho$ is the heat diffusivity of the blood, see Table 1 . The numerical 

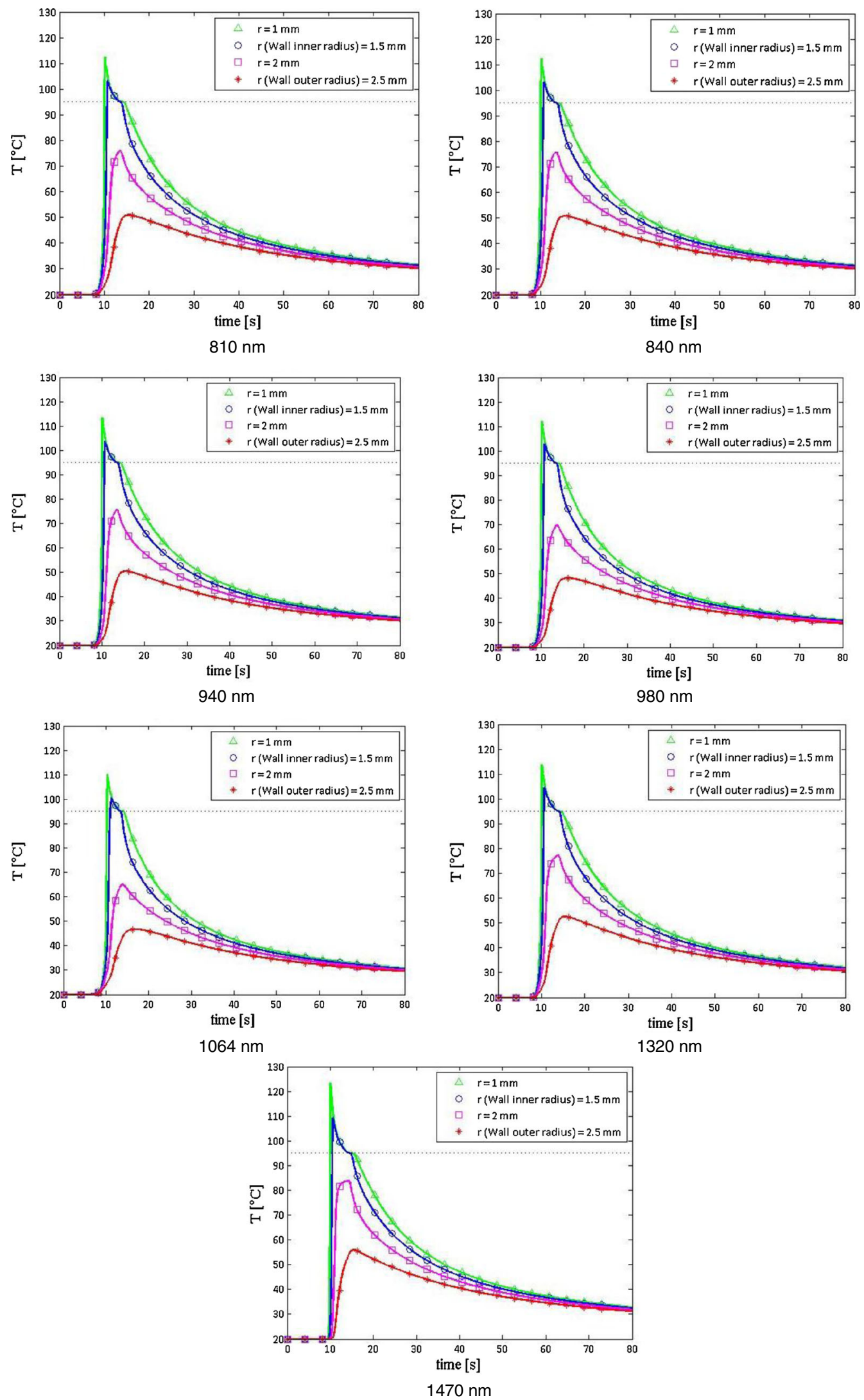

Fig. 3 Temperature profiles at various radial positions, as a function of wavelength and time for $P=15 \mathrm{~W}, v=2 \mathrm{~mm} / \mathrm{s}$, calculated at axial position $z=$ $40 \mathrm{~mm}$. The dashed line indicates the threshold temperature of $95^{\circ} \mathrm{C}$ 
implementation was validated by computing numerical solutions for several values of the grid size $\Delta r, \Delta \mathrm{z}$, and time step $\Delta t$. The accuracy is typically $0.05 \%$, defined as a maximum of absolute difference between numerical and analytical results divided by the analytical one. According to Nogotov [16], the spatial and temporal grid sizes need to satisfy the requirement

$\Delta t \leq \frac{(\Delta z)^{2}+(\Delta r)^{2}}{4 \alpha_{\max }}$

where $\alpha_{\max }$ is the maximum of heat diffusivity for the whole domain.

According to the results, the time step used in the calculations of EVLA was chosen as $0.05 \mathrm{~s}$, the radial, $\Delta r$, and axial, $\Delta z$, grid size as $10^{-4} \mathrm{~m}$. Grid independence was proven with some dedicated test runs.

\section{Numerical calculations}

Numerical calculation of the temperature field is done by an iterative process. Heat conductivity is artificially increased in the region $\left(r_{\text {fiber }}<r<r_{n}, z_{n}<z<z_{f}\right)$, where $z_{f}$ is the axial coordinate of the fiber tip, $n$ the number of time step. Parameters $r_{n}, z_{n}$ are taken such that

$$
\begin{aligned}
T_{\mathrm{n}-1} \mid & \mathrm{r}<\mathrm{r}_{\mathrm{n}}>95{ }^{\circ} \mathrm{C}, \\
\mathrm{z} & >\mathrm{z}_{\mathrm{n}}
\end{aligned}
$$

Where $T_{n-1}$ is the temperature that is calculated at the previous time step (Fig. 2). After each computation of the temperature field, $r_{n}$ and $z_{n}$ are recalculated. An example of three consecutive iterations of the temperature profile is presented in Fig. 2. At the edge of the "shoulder," the heat flux is constant, making

$\left.k_{\text {increased }} \frac{\delta T}{\delta r}\right|_{r<r_{n}}=\left.k \frac{\delta T}{\delta r}\right|_{r>r_{n}}$,

Thus, the inflection points in Fig. 2 are a consequence of the different coefficients of heat conduction around $T=95^{\circ} \mathrm{C}$. Temperature profile $T_{n-1}$ has also a second inflection point at $r=r_{\text {fiber }}$. At $t=10 \mathrm{~s}$, the fiber tip passes the observation point so the heat conductivity in region $r<r_{\text {fiber }}$ is equal to $k_{\text {fiber }}$.

\section{Results}

Several simulations of the temperature, $\mathrm{T}$, were performed for different wavelengths for the case where the laser power is $15 \mathrm{~W}$ and the fiber tip is pulled back with velocity $2 \mathrm{~mm} / \mathrm{s}$. Figure 3 presents the results showing temperature time histories at four radial positions.
Figure 3 shows that the temperature profiles inside the vein $(r \leq 1.5 \mathrm{~mm})$ have "shoulder" in the high-temperature region, i.e., $T>95^{\circ} \mathrm{C}$. When the fiber tip passes the axial position of observation (at $z=40 \mathrm{~mm}$, which is reached at $10 \mathrm{~s}$ after departure from $z_{0}=20 \mathrm{~mm}$ ), the temperature in the vein shows a very steep increase, and it exceeds the saturation temperature of blood $\left(100^{\circ} \mathrm{C}\right)$. As was mentioned before, if the temperature is higher than $95{ }^{\circ} \mathrm{C}$, the local heat conductivity is increased to $\mathrm{k}_{\text {increased }}=200 \mathrm{k}$. This creates the inflection point in the temperature profiles at $T=95^{\circ} \mathrm{C}$ (Fig. 3).

Laser treatment has been found to produce a thin carbonized layer covering the fiber tip that occurs at about $300{ }^{\circ} \mathrm{C}$. This black layer absorbs between 30 and $70 \%$ (with an average of $45 \%$ ) of the emitted light power and causes its high temperatures. The results for $T$ at zero radial position (the fiber tip) are presented in Table 3. Depending on the laser wavelength, it is around $950-1,100{ }^{\circ} \mathrm{C}$.

The temperature at the fiber tip depends weakly on the laser wavelength, which we attribute to differences in heat conduction from the thin carbonized layer. The blood temperature close to the fiber tip depends on the blood absorption coefficient, which, therefore, affects the temperature gradient between fiber tip and the boundary of the carbonized layer.

The maximum temperatures at the inner vein wall are also presented in Table 3. When the fiber tip passes the axial position at $40 \mathrm{~mm}$ (corresponding to $t=10 \mathrm{~s}$ ), the vein wall at $z=40 \mathrm{~mm}$ starts heating up. A short while later, when the fiber tip is slightly farther than $40 \mathrm{~mm}$, the temperature achieves its maximum value. The time where the temperature is maximal depends on the laser wavelength. For 810,840 , and $940 \mathrm{~nm}, T$ reaches its maximum at $10.75 \mathrm{~s}$. For $980 \mathrm{~nm}$, this is at $10.85 \mathrm{~s}$; for $1,064,1,320$ and $1,470 \mathrm{~nm}$, it is at 11.05 , 10.65 , and $10.6 \mathrm{~s}$, respectively.

The temperatures at the inner vein wall are weakly wavelength dependent in part because the wall absorption coefficients are wavelength dependent. However, heat diffusion strongly limits the variation in wall temperatures.

To additionally compare temperature profiles for different wavelengths, the following graphs were plotted. Figure 4

Table 3 Temperatures at the inner vein wall and in the carbonized layer, ${ }^{\circ} \mathrm{C}$

\begin{tabular}{lll}
\hline Laser wavelength, nm & $\begin{array}{l}T \text { at } r=0 \\
\text { at } t=10 \text { s (fiber tip) }\end{array}$ & $\begin{array}{l}T_{\max } \text { at inner } \\
\text { vein wall }\left(r_{i}\right)\end{array}$ \\
\hline 810 & 953 & 103 \\
840 & 954 & 103 \\
940 & 955 & 104 \\
980 & 953 & 102 \\
1,064 & 952 & 101 \\
1,320 & 955 & 104 \\
1,470 & 1,104 & 109 \\
\hline
\end{tabular}


Fig. 4 Temperature profiles per wavelength depends on time at different radial positions, $P=15 \mathrm{~W}, v=2 \mathrm{~mm} / \mathrm{s}$
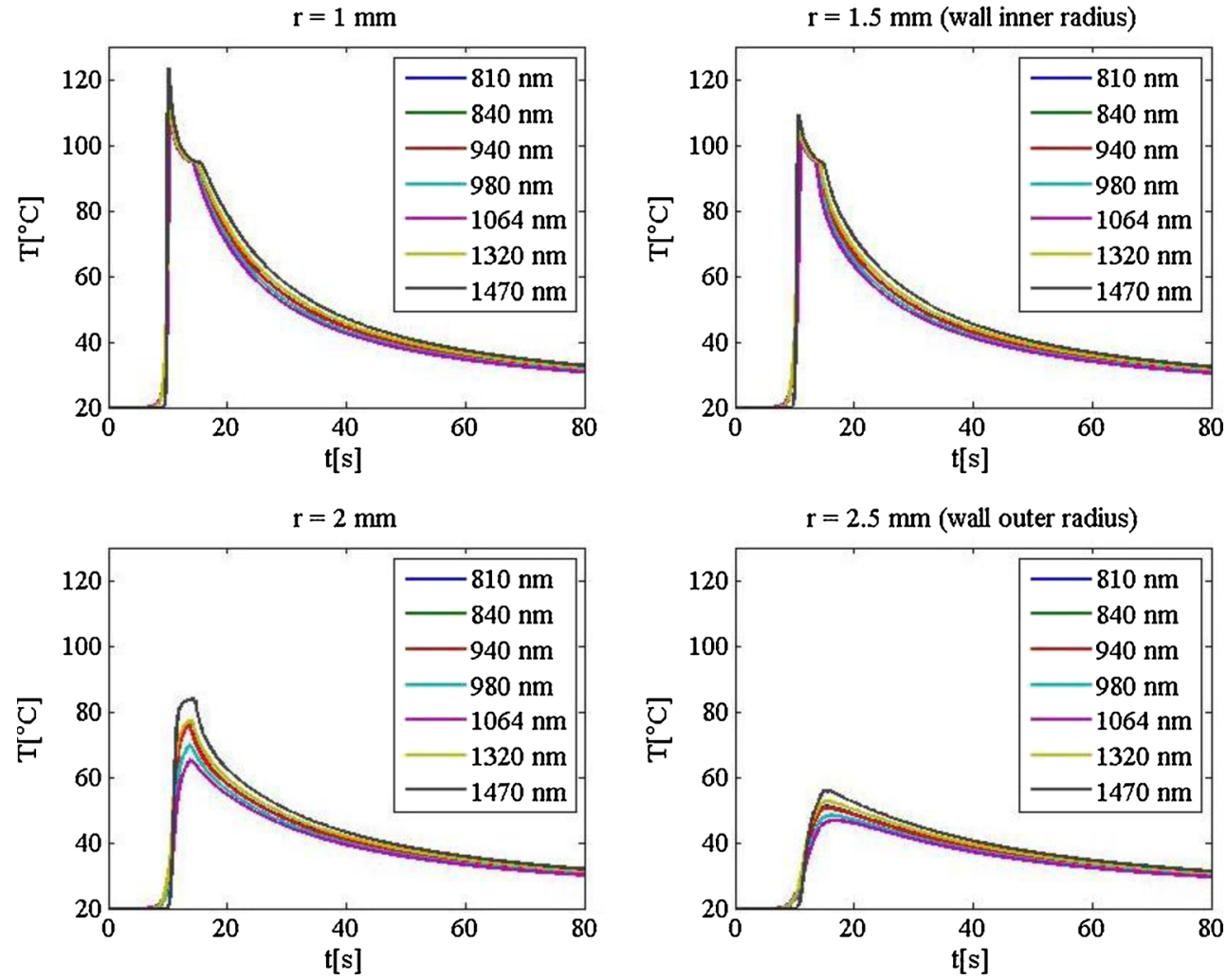

presents the temperature-time evolution at different radial positions, and Fig. 5 present the radial evolution of temperature at different times.

Figure 4 show that the laser wavelength does not significantly influence the temperature-time history inside the vein. Thus, from Table 3 and Figs. 4 and 5, the conclusion can be drawn that the temperature profile inside the vein is virtually wavelength independent. It agrees very well with a recent experimental study [5] where wavelength independence was found.

Fig. 5 Temperature profiles per wavelength depends on the radius at different times, $P=15 \mathrm{~W}, v=$ $2 \mathrm{~mm} / \mathrm{s}$. Dashed lines indicate inner and outer vein walls respectively
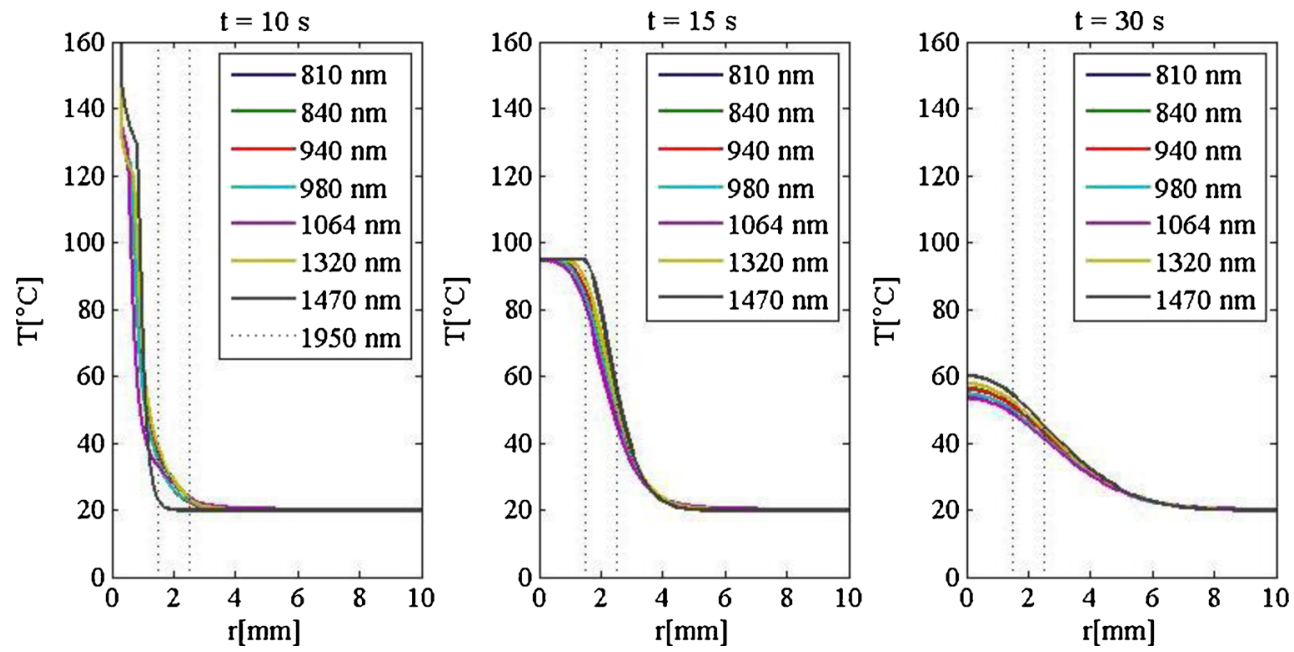

Finally, we show that black body radiation from the black carbonized layer has a radiative heat transfer that is negligibly small compared to heat diffusion, as is easily seen as follows. The power, $P_{r}$, radiated from the hot layer is given as follows:

$P_{r}=\sigma T^{4} A$,

Where $\sigma=5.6704 \cdot 10^{-8} \frac{W}{m^{2} K^{4}}$ is the Stefan-Boltzmann constant, $T$ the temperature of the hot layer in degrees Kelvin, assumed to be $1,100 \mathrm{~K}$ here, and $A$ the surface area of the 
carbonized layer, assumed equal to the area of the fiber tip with radius $0.3 \mathrm{~mm}$. The black body radiation

$P_{r}=5.6704 \cdot 10^{-8} \cdot 1100^{4} \cdot \pi \cdot\left(0.3 \cdot 10^{-3}\right)^{2}=0.0235 \mathrm{~W}$

is much smaller than the laser power $P=15 \mathrm{~W}$.

The wavelength at which the intensity of the radiation produced by a black body is at a maximum, $\lambda_{\max }$, is a function only of the temperature.

$\lambda_{\max }=\frac{b}{T}$,

Where $b=2.8977721 \cdot 10^{-3}$ is Wien's displacement constant. So, for the black body at $T=1,100 \mathrm{~K}, \lambda_{\max }$ is equal to $2,630 \mathrm{~nm}$. The absorption coefficient corresponding to the wavelength of $2,630 \mathrm{~nm}$ is around $100 \mathrm{~mm}^{-1}$, albeit it over a small waveband only. To evaluate the contribution of the radiative heat transfer from the carbonized layer, the black body radiation was simulated with the parameters discussed above by setting the laser power at $0.02 \mathrm{~W}$ instead of $15 \mathrm{~W}$. As a result of the simulation, it is established that the black body radiation does not influence the temperature distribution. It proves that the radiative heat transfer from the black carbonized layer is negligibly smaller compared to heat diffusion.

\section{Discussion}

The presented EVLA optical-thermal mathematical model improves Mordon's model [8] by including black body radiation and the thin layer of carbonized blood on the fiber tip, which absorbs between 30 and $70 \%$ of the laser light emitted out of the fiber. The predicted temperatures of the layer, between 950 and $1,100{ }^{\circ} \mathrm{C}$, correspond well to reported experimental results $[1-4,9,10]$ and also other predicted temperatures are in agreement with experiments [5], reconfirming that heat diffusion from this hot layer is a major EVLA mechanism [5, 12].

The model also confirms previous suggestions that wavelength plays a minor role in EVLA, contrary to the suggestions by companies.

EVLA procedures produce boiling bubbles emerging from pores within the hot fiber tip. These steam bubbles detach from the fiber tip and transport heat to the distal location where condensation occurs. To account for heat transfer by these boiling bubbles, the thermal conductivity was artificially increased 200 times, in that part of the blood volume where the temperature is close to or above the saturation temperature of $100{ }^{\circ} \mathrm{C}$. In this way, energy is conserved while temperatures in the blood seldom exceed $100{ }^{\circ} \mathrm{C}$. In our opinion, this procedure is physically more realistic than the simple cutting off of the temperature to $100{ }^{\circ} \mathrm{C}$ when temperatures exceed $100{ }^{\circ} \mathrm{C}$, because the energy associated with these temperatures in excess of $100{ }^{\circ} \mathrm{C}$ is now being destroyed, implying conservation of energy is not assured. For the time being, this approach allowed us to avoid the complex simulation of fluid flow, phase change, and steam bubble transport.

In conclusion, our simulations confirm our previous hypothesis that temperature profiles are virtually independent of the laser wavelength, and that the thin carbonized blood layer that is always present on the fiber tip reaches temperatures of around $1,000^{\circ} \mathrm{C}$.

\section{References}

1. Weiss RA (2002) Comparison of endovenous radiofrequency versus $810 \mathrm{~nm}$ diode laser occlusion of large veins in an animal model. Dermatol Surg 28:56-61

2. Zimmet S, Min RJ (2003) Temperature changes in perivenous tissue during endovenous laser treatment in a swine model. J Vasc Interv Radiol 14:911-915

3. Fan C-M, Anderson RR (2008) Endovenous laser ablation: mechanism of action. Phlebology 23:206-213

4. Disselhoff BCVM, Rem AI, Verdaasdonk RM, der Kinderen DJ, Moll FL (2008) Endovenous laser ablation: an experimental study on the mechanism of action. Phlebology 23:69-76

5. van den Bos R, van Ruijven PW, van der Geld CW, van Gemert MJ, Neumann HA, Nijsten T (2012) Endovenous simulated laser experiments at 940 and 1,470 nm suggest wavelength independent temperature profiles. Eur J Vasc Endovasc Surg 44:77-81

6. Vuylsteke ME, Mordon SR (2012) Endovenous laser ablation: a review of mechanisms of action. Ann Vasc Surg 26:424 433

7. Van Gemert MJC, van derGeld CWM, Bruijninckx CMA, Verdaasdonk RM, Neumann HAM (2012) Comment to Vuylsteke ME and Mordon SR. Endovenous laser ablation: a review of mechanisms of action, Ann Vasc Surg 26:424-433. Ann Vasc Surg 2012; 26:881-883

8. Mordon SR, Wassmer B, Zemmouri J (2007) Mathematical modeling of 980 and 1,320-nm endovenous laser treatment. Lasers Surg Med $39: 256-265$

9. Meissner OA, Schmedt C-G, Hunger K, Hetterich H, Sroka R, Rieber J, Babaryka G, Steckmeier BM, Reiser M, Siebert U, Mueller-Lisse U (2007) Endovascular optical coherence tomography ex vivo: venous wall anatomy and tissue alterations after endovenous therapy. Eur Radiol 17:2384-2393

10. Verdaasdonk RM, Holstege FC, Jansen ED, Borst C (1991) Temperature along the surface of modified fiber tips for Nd:YAG laser angioplasty. Lasers Surg Med 11:213-222

11. van den Bos RR, Kockaert MA, Neumann HAM, Bremmer RH, Nijsten T, van Gemert MJC (2009) Heat conduction from the very hot fiber tip contributes to endovenous laser ablation of varicose veins. Lasers Med Sci 24:247-251, Erratum 2009; 24:679

12. Amzayyb M, van den Bos RR, Kodach VM, de Bruin DM, Nijsten T, Neumann HAM, van Gemert MJC (2010) Carbonized blood 
deposited on fibers during 810, 940, and 1,470 nm endovenous laser ablation: thickness and absorption by optical coherence tomography. Lasers Med Sci 25:439-447

13. Star WM (2011) Diffusion theory of light transport. In Welch AJ and van Gemert MJC (eds) Optical-thermal response of laser-irradiated tissue. 2nd edition. Springer, Dordrecht, The Netherlands, Chapter 6, Equation (6.104), page 183
14. Kuenstner JT, Norris KH (1994) Spectrophotometry of human hemoglobin in the near infrared region from 1,000 to $2,500 \mathrm{~nm}$. J Near Infrared Spectrosc 2:59-65

15. Carslaw HS, Jaeger JC (1986) Conduction of heat in solids, 2nd edition, reprint. Clarendon, Oxford

16. Nogotov EF (1978) Applications of numerical heat transfer. McGraw-Hill, New York 